\title{
Effect of Collagen -Activated Platelet-Rich Plasma to Synthesis of Extracellular Matrix in Fibroblast of Periodontal Ligament
}

\author{
Kwartarini Murdiastuti*, Pati Tangsupati \\ Department of Periodontology, Faculty of Dentistry, Universitas Gadjah Mada, Yogyakarta, \\ Indonesia \\ *Email: kmurdiastuti@ugm.ac.id
}

\begin{abstract}
The aim of this study was to investigate the activation effect of PRP using collagen to the synthesis of extracellular matrix in fibroblasts of the periodontal ligament. The PRP from the human donor was activated using collagen (collagenactivated PRP) and compared with inactivated PRP as a control group. The groups have measured the deposit of extracellular matrix during the three and seven days for observation. The collagen-activated PRP resulted in a more significant release of extracellular matrix compared with control group. And, there were substantial differences of extracellular matrix deposit in the three and seven days for observation. We obtained that the use of collagen on PRP as an alternative to stimulate platelet-associated growth factors and give greater extracellular matrix synthesis. The polypeptide growth factor (PGFs) is a biological mediator in periodontal regeneration. These materials are desirable because of regulatory effects on proliferation and differentiation of bone cells and connective tissues. Platelet Derived Growth Factor (PDGF) and Transforming Growth Factor - $\beta$ (TGF - $\beta$ ) are the most potent stimulators of PGFs for fibroblasts in cell migration, and proliferation as well as in the synthesis of extracellular matrix. It is vital in the process of wound healing. Platelet - Rich Plasma could improve the activity of extracellular matrix synthesis of periodontal ligament fibroblasts. The addition of rat tail collagen in PRP did not affect the increase in extracellular matrix synthesis activity of periodontal ligament fibroblasts. Addition collacure collagen sponge on the PRP was affected on the growing movement of the extracellular matrix of periodontal ligament fibroblasts.
\end{abstract}

Keywords: collagen-activated PRP, synthesis of extracellular matrix, fibroblasts periodontal ligament

\section{Introduction}

Periodontal disease is a pathological condition characterized by destruction of periodontal tissue which includes the gingiva, alveolar bone, periodontal ligament and cementum [1]. Treatment of periodontal surgery is the form of regenerative procedure, which the goal is to improve the structure and function of the periodontium tissue. Regeneration is a reproduction or reconstruction of body parts that are damaged or missing, including the form and function of tissues. The periodontal regenerative procedure is intended to improve the structure gingiva, alveolar bone, cementum and periodontal ligament that is strong enough to support teeth [2]. Wound 
healing is a dynamic process consisting of continuity four phases: homeostasis, inflammation, proliferation, and remodeling tissue or resolution. Regenerative process is mediated by the presence of fibroblasts, new blood vessels and chronic inflammatory cells in areas of injury. In the phase of proliferation, migration, infiltration, proliferation, and differentiation of the cells is an essential process for the formation of new tissue and lead to wound closure. Increasing the number of fibroblasts in the wound area is a combination of proliferation and migration, growth caused by cytokine produced by macrophages and lymphocytes. Fibroblasts are the main elements in the repair process for the formation of structural proteins that play a role in tissue formation. The method of migration and proliferation of fibroblasts is an essential process in the creation of granulation tissue and wound closure. Migration periodontal ligament fibroblasts to root surface is critical to the regeneration of the periodontal ligament, as in the rehabilitation of the periodontal ligament of rapid migration of cells on the root surface will play a significant role in the reattachment of the periodontal ligament fibroblasts during repairs [3]. Wound healing process is an essential component of collagen formation, immediately after the injury, collagen fibers can get into the blood causing platelet aggregation and activation and release chemotactic factors that initiate the wound healing process. Fragments of collagen release collagenase leucocytic to attract fibroblasts to the area of injury. Furthermore, the collagen became the foundation for the formation of a new extracellular matrix (ECM) [4].

Platelet-Rich Plasma (PRP) is a preparation of autologous platelet high concentration obtained by centrifugation of autologous blood [5]. Platelets release Platelet Derived Growth Factor (PDGF), Transforming Growth Factor- $\beta$ (TGF- $\beta$ ), Vascular Endothelial Growth Factor (VEGF), Basic fibroblast growth factor (bFGF), Epidermal Growth factor (EGF), Insulin Growth Factor (IGF), Platelet-Derived Endothelial Cell Growth Factor (PDECGF), Platelet Derived Angiogenesis Factor (PDAF) and Fibroblast Growth Factor (FGF), which play a significant role in the process of migration, proliferation and differentiation [6].Platelet Rich Plasma can stimulate spending Polypeptides Growth Factor (PGF) which can regulate biological event in the wound healing process such as cell adhesion, cell migration, proliferation, differentiation and morphogenesis of tissues, including the synthesis of ECM as a mediator biological process periodontal regeneration. And PGF a stimulator for fibroblast in cell migration, mitogenesis and proliferation as well as in the synthesis of the matrix, it is important in the wound healing process [7]. So growth factor in PRP can stimulate the fibroblast of periodontal ligament to the combination of ECM and indicators that will be observed by an increase in the activity to produceECM by periodontal ligament fibroblasts which are progenitor cells for soft tissue and hard tissue periodontal.

The use PRP in the treatment of periodontal disease is the finding that continues to be developed because of its ability to secrete a growth factor that is necessary for the process of tissue regeneration. Growth factor will be much removed from the granules during platelet activation. Platelet activator is widely used for this is the cow thrombin. However, some side effects have been reported using cow thrombin, which is a reaction to the formation of antibodies against thrombin, prothrombin, 
factor V and cardiopilin clinically can cause bleeding after surgery and in experimental animals cause an autoimmune syndrome similar lupus disease [7]. Another deficiency use of thrombin as the activator PRP cow is to inhibit proliferation and cell viability in vitro studies. Also, serine proteases including thrombin can degrade growth factor. In vitro studies known cow thrombin was inhibiting the migration of fibroblasts and reduces clot strength [4].

Collagen is an alternative to replace thrombin as the activator of PRP because of involvement in the intrinsic clotting cascade and is widely used as a biomaterial. Fufa et al. use collagen type I as a clotting agent and activator of platelets in PRP instead of bovine thrombin, by measuring the level of expenditure of TGF- $\beta 1$, PDGF and VEGF of both types of clots for few days. The cumulative release of TGF- $\beta 1$ on PRP is higher when collagen is used as an activator. Activator of collagen show sustained release of cytokines during few days. How to collagen activates platelets are platelets should interact with the collagen and then activated through receptor GP VI. In a collagen wound healing often act as first platelet activators, by forming a monolayer of platelets to collagen injured area [4].

In this study, PRP will be added collagen to activate the release of growth factors from its granular, because collagen is a protein that is naturally involved in the liberation of growth factor from platelet granules in the human. This study aimed to investigate the activation effect of PRP using collagen on the synthesis of extracellular matrix in fibroblasts of the periodontal ligament.

\section{Materials and Methods}

This research is a pure experimental research, with variables: (a). Effect Variable: This type of treatment: Platelet Rich Plasma (PRP) andcollagen-activatedPRP andincubated for 24, 48, 72 and 168 hours, after which lysate was taken and time Observations 12, 24, 48 and 72 hours (b). Affected Variable: synthesis of extracellular matrix in fibroblasts of the periodontal ligament.

Two kinds of collagen used in this study are pure collagen from rat tail (RT Collagen) solution (commonly used in basic research) and Collacure Collagen Sponge from bovine tendon Achilles (often used dermatologist to treat the cosmetic problem).

Then cultured fibroblast cells as the study samples were divided into seven groups, one unstimulated group as the negative control, one group was stimulated with PRP lysate alone, and four other groups each stimulated with PRP + RT collagen lysates that had been incubated for 24,48,72 and 168 hours. The last group Collacure collagen-activated PRP at 24 hours. Calculation of matrix deposition conducted on day 3 and day 7 .

The study was conducted in the following procedures: (i) the culture of fibroblasts, cells periodontal ligament taken from the root surfaces of the teeth using a blade \# 15, the ligament was made in the middle third of the tooth root, ligament shredded and then cultured using culture media Dubelco's Modification of Eagle's Medium (DMEM), added a supplement of $10 \%$ FBS, penicillin-streptomycin 2\%, $0.5 \%$ fungizone then put in a $\mathrm{CO} 2$ incubator at a temperature of $37^{\circ} \mathrm{C}$ [8], (ii) preparation of donor blood PRP supernatant was taken as $100 \mathrm{ml}$, then entered into a tube containing $1 \mathrm{ml}$ of $3.8 \%$ sodium citrate, and then inverted slowly so that both 
of them mixed. Each tube was centrifuged for ten minutes at $2400 \mathrm{rpm}$ rotation. This centrifuge produced two layers, for the top layer contained Platelet Plasmaand bottom layer consisted of red blood cell. Platelet Plasmalayer was taken using a long cannula and water-intake cannula inserted into tubes without anticoagulant. Tubes were centrifuged for 15 minutes at $3600 \mathrm{rpm}$ rotation. This second centrifugation would produce two layers; the top layer was a Platelet Poor Plasma (PPP) $2 / 3$ and $1 / 3$ bottom layer was the PRP. Platelet-rich plasma was separated from PPP with isolation kit [9]. (iii) Two kinds of collagen used in this study are pure collagen from rat tail solution (commonly used in basic research) and Collacure (Collagen Sponge) from bovine tendon Achilles (often used dermatologist to treat a cosmetic problem). (iv) Briefly, fibroblasts with a density of 10,000 cells / 35-mm, next day, the culture medium were replaced with medium DMEM serum-free(v) each Collagen-activated PRP was obtained by mixing the PRP with collagen, the volume ratio is 1: 17. (vi) PRP was activated by collagen and incubated for $24,48,72$ and 168 hours, after which lysate was taken. (vi) The samples were divided into seven groups and given different treatments.One group unstimulated groupwas as a negative control, the second group was only stimulated by PRP lysate, and four other groups, the groups were encouraged by rat tail collagen-activated PRP which had been incubatedduring 24,48,72 and 168 hours. The last group wasstimulated by collacure collagen-activated PRP which had been incubated for 24 hours.Each group in 10 wells of microplate 24 wells and according to the hours of incubation the cells washed with FBS.(vii)All groups were measured the deposit of extracellular matrix by MTT assay after three and seven days.

\section{Results}

This in vitro study was starting to see the effects of collagen-activated PRP to the extracellular matrix synthesis activity of fibroblasts of periodontal ligament (progenitor cells). In the cascade of periodontal tissue regeneration and reconstruction, the synthesis of the extracellular matrix is an essential part. The data of this research was the absorbance value of each sample was read using a plate reader spectrophotometer technique. The resultwas listed in Table 1 as follows:

Table 1. The mean and standard deviation of the synthesis of ECM of the fibroblasts of periodontal ligament after PRP activated with Collagen

\begin{tabular}{|l|c|c|}
\hline \multirow{2}{*}{ Group } & \multicolumn{2}{|c|}{ Observation time } \\
\cline { 2 - 3 } & Days-3 (n=42) & Days-7 (n=42) \\
\hline PRP & $0,1297 \pm 0,0165$ & $0,3553 \pm 0,2179$ \\
\hline Negative control (C -) & $0,0797 \pm 0,0053$ & $0,0972 \pm 0,0064$ \\
\hline Lysate PRP+ RT Collagen 24h (P1) & $0,1228 \pm 0,0057$ & $0,2377 \pm 0,0470$ \\
\hline Lysate PRP+RT Collagen 48h (P2) & $0,1800 \pm 0,0731$ & $0,3195 \pm 0,0493$ \\
\hline Lysate PRP+RT Collagen 72h P(3) & $0,1713 \pm 0,0303$ & $0,2802 \pm 0,0991$ \\
\hline Lysate PRP+RT Collagen 168h (P4) & $0,1505 \pm 0,0337$ & $0,2540 \pm 0,0437$ \\
\hline Lysate PRP+Collacure Collagen 24h (P5) & $0,1527 \pm 0,0142$ & $1,6395 \pm 0,8819$ \\
\hline
\end{tabular}


Table 1 showed that the lowest mean of extracellular matrix deposit was in the (-) control group after three days observation $(0,0797 \pm 0,0053)$. The highest one was collared collagen-activated PRP for 24 hoursand after seven days observation $(1,6395 \pm 0.8819)$. This was evident in the following Fig 1 and Fig 2.

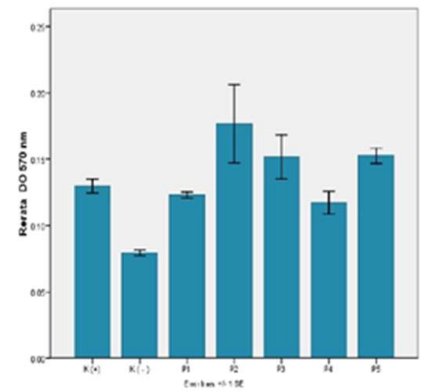

Fig 1. The extracellular matrix synthesis means in treatment group after three

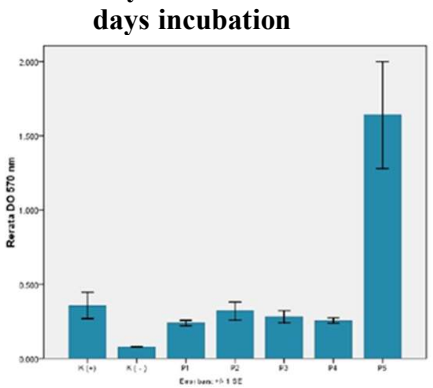

Fig 2. The extracellular matrix synthesis implies in treatment group after seven days incubation

It could be seen the lowest mean and standard deviation of the ECM, after 3 and seven days of incubation was in the negative control group (the group unstimulated cells). Then beginning to increase was started from the positive control which the group of fibroblasts were stimulated with PRP andP1 and P2 groupswhich fibroblasts were stimulated with rat tail collagen-activated PRP and incubated for 24 and 48 hours at both observation times. At P3 and P4 group, the mean value started to move down, which the group of fibroblasts were stimulated with rat tail collagenactivated PRP from 72 to 168 hours of incubation. The average amount increased again in the P5 group that fibroblasts were boosted by Collacure and incubated for 24 hours. However,the ECM production in the P5 increased almost eight-fold (8x) compared to the other groups.

Prerequisites use Two Ways ANOVA test was the data to be normally distributed, therefore before the normality test using the Kolmogorov Smirnov test had to be done on all data readings plate reader. 
Table 2. The result of normality test using Kolmogorov Smirnovtest in the treatment groups and after 3 and seven days of observation time

\begin{tabular}{|l|c|c|}
\hline \multirow{2}{*}{\multicolumn{1}{c|}{ Group }} & \multicolumn{2}{c|}{ Incubation time } \\
\cline { 2 - 3 } & 3 days & 7 days \\
\hline PRP & 0.687 & 0,978 \\
\hline Negative control (C -) & 0.448 & 0.462 \\
\hline Lysate PRP+ RT Collagen 24h (P1) & 0.516 & 0.862 \\
\hline Lysate PRP+RT Collagen 48h (P2) & 0.906 & 0.963 \\
\hline Lysate PRP+RT Collagen 72h P(3) & 0.392 & 0.894 \\
\hline Lysate PRP+RT Collagen 168h (P4) & 0.540 & 0.673 \\
\hline Lysate PRP+Collacure Collagen 24h (P5) & 0.567 & 0.618 \\
\hline
\end{tabular}

Kolmogorov Smirnov normality test results for all groups indicated that the data were normally distributed ( $\mathrm{p} \geq 0.05$ ) so that the analysis can be continued by using parametric analysis Two-ways ANOVA, with post hoc Least Significance Different test (LSD).

Table 3. Results of Post Hoc Least Significance Test Different (LSD) Extracellular Matrix Synthesis Incubation After 3 Days

\begin{tabular}{|l|r|r|r|r|l|l|l|}
\hline \multicolumn{1}{|c|}{ Group } & PRP & C (-) & P1 & P2 & \multicolumn{1}{|c|}{ P3 } & \multicolumn{1}{|c|}{ P4 } & \multicolumn{1}{|c|}{ P5 } \\
\hline PRP & & $0.014^{*}$ & 0.727 & $0.014^{*}$ & $0.039^{*}$ & 0.291 & 0.245 \\
\hline Negative control (C -) & & & $0.032^{*}$ & $0.000^{*}$ & $0.000^{*}$ & $0.001^{*}$ & $0.001^{*}$ \\
\hline Lysate PRP+ RT Collagen 24h (P1) & & & & $0.006^{*}$ & $0.017^{*}$ & 0.163 & 0.134 \\
\hline Lysate PRP+RT Collagen 48h (P2) & & & & & 0.658 & 0.138 & 0.168 \\
\hline Lysate PRP+RT Collagen 72h P(3) & & & & & & 0.291 & 0.343 \\
\hline Lysate PRP+RT Collagen 168h (P4) & & & & & & & $0.002^{*}$ \\
\hline Lysate PRP+Collacure Collagen 24h (P5) & & & & & & & \\
\hline
\end{tabular}

Results of Post Hoc Least Significance Test Different (LSD) was presented in Table 4 showed a comparison between the negative control group and positive group and alsonegative control group with the other treatment groups had significant differences $(\mathrm{p}<0.05)$.

Table 4. Results of Post Hoc Least Significance Test Different (LSD) Extracellular Matrix Synthesis After Incubation 7 days

\begin{tabular}{|l|r|r|r|r|r|r|c|}
\hline \multicolumn{1}{|c|}{ Group } & PRP & C (-) & P1 & P 2 & P 3 & P 4 & P 5 \\
\hline PRP & & 0.178 & 0.565 & 0.861 & 0.713 & 0.620 & $0.000^{*}$ \\
\hline Negative control (C -) & & & 0.433 & 0.240 & 0.323 & 0.898 & $0.000^{*}$ \\
\hline Lysate PRP+ RT Collagen 24 J (P1) & & & & 0.689 & 0.835 & 0.939 & $0.000^{*}$ \\
\hline Lysate PRP+RT Collagen 48h (P2) & & & & & 0.847 & 0.748 & $0.000^{*}$ \\
\hline Lysate PRP+RT Collagen 72h P(3) & & & & & & 0.848 & $0.000^{*}$ \\
\hline Lysate PRP+RT Collagen 168h (P4) & & & & & & & $0.000^{*}$ \\
\hline LysatePRP+Collacure Collagen 24h (P5) & & & & & & & \\
\hline
\end{tabular}


Results of Post Hoc Least Significance Test Different (LSD) on the synthesis of ECM among groups after an incubation period of 7 days showedthere were no significant differences between one group to another $(p>0.05)$. Exceptions to the P5 group that fibroblasts were stimulated with Collacure collagen, comparison with all other groups showed a very significant difference $(p=0.000)$. That means the activation by rat tail collagen showed no effect in increasing the ECM synthesis after incubation for seven days. In contrast, the addition of collagen from bovine tendon Achilles had a significant impact in improving the ECM production. This could be related to the biocompatibility of biomaterials derived from bovine is higher than that obtained from the rat. Differences in extracellular matrix synthesis based on the observation time could be achieved by using independent t-test trials, the results were presented in Table 5 .

Table 5. Results of Independent Sample t-test betweenobservation time in all the groups.

\begin{tabular}{|l|c|c|}
\hline \multicolumn{1}{|c|}{ Group } & n & Significance Level (p) \\
\hline PRP & 12 & $0,030^{*}$ \\
\hline negative control (C -) & 12 & $0,050^{*}$ \\
\hline Lysate PRP+ RT Collagen 24 J (P1) & 12 & $0,000^{*}$ \\
\hline Lysate PRP+RT Collagen 48h (P2) & 12 & $0,043^{*}$ \\
\hline Lysate PRP+RT Collagen 72h P(3) & 12 & $0,026^{*}$ \\
\hline Lysate PRP+RT Collagen 168h (P4) & 12 & $0,000^{*}$ \\
\hline Lysate PRP+Collacure Collagen 24h (P5) & 12 & $0,000^{*}$ \\
\hline
\end{tabular}

All groups indicated that there was a significant difference between observation time in each group $(\mathrm{p}<0.05)$. An increase in the numbers of extracellular matrix synthesis in all groups when compared to the observations after 3 with seven days.

\section{Discussion}

Extracellular matrix synthesis in this study was measured by the spectrophotometer techniques and read at a wavelength of $570 \mathrm{~nm}$, the numbers were read from the plate reader absorbance value representing the colour density of each sample was given reagent Sirius red. Once given the sample-reagent would change colour. Change colour was then read with a spectrophotometer plate reader light intensity passing through the samples. Observation data trends on day 3 after incubation showed the lowest mean value of the negative control group, the group of unstimulated fibroblasts, began to increase in the positive control group, the group of fibroblasts were stimulated with PRP and incubated for 24 hours and in group P1 namely the group by rat tail collagen-activated PRP and incubated for 24 hours. Mean value P2 group peaked in the group ofrat tail collagen-activated PRP and incubated for 48 hours. At P3 and P4 group, the average value started to move down, which was the group of rat tail collagen-activated PRP and incubated for 72 hours and 168 hours. Average cost increased again in the P5 group Collacure collagen-activated PRP and incubated for 24 hours. The movement of data on the observation after seven days showed a similar pattern with views on the three days, but the extracellular matrix 
production in the P5 increased almost eightfold compared to the other groups.In observation for 3 days, as described in Table 2, found a comparison between all treatment groups compared with the negative control group, $\mathrm{p}<0.05$, meaning there are significant differences between the negative control group which was not stimulated by the PRP group and PRP wereactivated by either collagen or added without the addition of collagen. This suggested that PRP can increase the number of extracellular matrix synthesis in periodontal ligament fibroblasts. PRP derived from concentrated platelets, identified as one of the mediators have many growth factors that are important at all stages of the cell cycle and can stimulate chemotaxis and production of extracellular matrix proteins. Each growth factor has a regulatory function on the cellular level and is involved in the process of tissue regeneration. Growth Factor incurred in the process of platelet degranulation, such as transforming growth factor $\beta$ - 1 (TGF- $\beta 1$ ); Derived Platelet Growth Factor - B (PDGF-B), PDGF - A, PDGF AB; Fibroblast Growth Factor (FGF); Insulin Growth Factor (IGF), vascular endothelial growth factor (VEGF)6. While Harrison et al. (2011)4 proved that the release of TGF - $\beta 1$ PRP three times as much, PDGF - AB 2.5 times more, and VEGF 5 times more compared to whole blood. While according to Tozum et al. (2003) the preparation of PRP can be used to increase the local concentration of active PDGF - $\beta$ and TGF up more than $300 \%$.

Results of Table 3 meant that the addition of rat tail collagen showed no effect in increasing the extracellular matrix synthesis after observation for seven days. In contrast, the addition of collagen from bovine tendon Achilles a significant impact in raising the extracellular matrix production by periodontal ligament fibroblasts. This could be related to the biocompatibility of biomaterials derived from cows is higher than that obtained from mice.

Effect of collagen to increased extracellular matrix synthesis may occur from different pathways. First, collagen as an activator of PRP can stimulate spending growth factor that directly stimulates fibroblasts to increase production of extracellular matrix. Second, collagen stimulates the release of growth factors from platelet granules that will increase proliferation of fibroblasts as the cells that produce extracellular matrix. Growth factors released from platelet granules through a process of molecular interaction of activated platelets at the time, so the process of platelet activation plays a crucial in the process of tissue regeneration, collagen used in this study to enable platelet degranulation process. Collagen is a protein molecule that is naturally triple helix involved in the cascade of aggregation and degranulation of platelets in the body $[6,10]$. Platelet activation is a complicated process including a series of feedback and crosstalk between different pathways. Signaling pathways between the various specific platelet receptors simultaneously stimulate platelet shape change, secretion of growth factors and other molecules of the granule, and eventually induce an inside-out signaling process that activates integrin binding to its ligand $\alpha 2 \mathrm{~b} \beta 3$. This bond then leads to platelet aggregation and adhesion and outside-in signaling triggers which cause the secretion of granule contents, stabilize adhesion, platelet aggregation and clot. The process of platelet activation induced by collagen begins to bond with receptors GP VI collagen, which binds with FcR $\gamma$, this bond produces a series of intracellular signals, on the one hand, activates integrins $\alpha 2 b \beta 3$ which then binds with other collagen molecules. Association of collagen 
with the two primary receptors then induce intracellular signaling cascade that leads to the release of calcium and activation of protein kinase $\mathrm{C}(\mathrm{PKC})$, both responsible for the platelet response to the signal sequence, the withdrawn of platelet aggregation and release meant a granule contents through the process exocytosis [11]. The pattern of growth factor release from platelet granules can be different, depending on the activator used. Harrison et al. (2011)4 found PRP were activated with collagen continuously secrete growth factors for seven days and spend more TGF $\beta$ - 1 during the seven days of observation compared with that activated by thrombin. While the total amount of PDGF - AB and VEGF were issued relatively the same in both the PRP is activated by thrombin and activated by collagen within seven days.

PRP was activated with collagen to form a more stable clot to retract. Fufa et al., (2008)7 states PRP clots are stimulated with collagen view by $50 \%$ within the first 24 hours. After it is stable up to ten days, while the PRP clot that is activated by thrombin clot retracted up to $90 \%$ within the first 24 hours, and destroyed in serum after five days. Resistant characteristics of clot retraction force are needed in tissue engineering applications, where the premature degradation and clot retraction excessive charging and can inhibit wound healing. This aspect is essential for the PRP clot functions as a scaffold wound healing. This could be related to the levels of growth factors and release profiles of platelet granules, that the PRP is activated with collagen to induce spending TGF $\beta-1$ in more significant quantities. Transforming Growth Factor $\beta$ - 1 (TGF $\beta-1$ ) acts as a stimulator of the formation of the extracellular matrix, through the resultant of 4 process work TGF $\beta-1$, namely: 1) the stimulation of the synthesis of extracellular matrix components, 2) the stimulation of the synthesis of integrins, i.e. membrane receptors that allow cells extracellular recognize specific molecules in the basement membrane and other cells, 3) inhibits the synthesis of protease inhibitors which functions to break down the extracellular matrix, and 4) reduce the synthesis of ECM degrading protease enzyme that breaks down the extracellular matrix [4]. Table 5 showed an increase in the numbers of extracellular matrix synthesis in all groups when compared to the observations after three days of incubation with view after seven days incubation. This showedthat after three days of incubation, the activity of extracellular matrix synthesis by periodontal ligament fibroblasts increased. It could also be caused by an increase in the number of fibroblasts that produce extracellular matrix. This is in line with the explanation Guo \& DiPietro (2010)15 that the wound healing process, four phases take place continuously and overlap, starting from the period of hemostasis, inflammatory phase, the proliferative phase and remodeling phase. In this process, the extracellular matrix synthesis began in the proliferative phase on day three and continued to increase until well into the remodeling phase on day 21 .

\section{Conclusion}

Based on the research that had been done, it could be concluded as follows: PlateletRich Plasma could improve the activity of extracellular matrix synthesis of periodontal ligament fibroblasts; The addition of rat tail collagen in PRP did not affect 
the increase in extracellular matrix synthesis activity of periodontal ligament fibro-

blasts; Addition collacure collagen sponge on the PRP effected on the increased activity of extracellular matrix of periodontal ligament fibroblasts.

\section{Acknowledgement}

Grants-in-aid supported this work for scientific research from the Minister of Education and Culture, Directorate General of Higher Education (DGHE, Indonesia).

\section{References}

1. Shue L, Yufeng Z, Mony U. Biomaterials for periodontal regeneration: a review of ceramics and polymers. Biomatter. 2012 Oct 1;2(4):271-7.

2. Bosshardt DD, Sculean A. Does periodontal tissue regeneration really work? Periodontology 2000. 2009 Oct 1;51(1):208-19.

3. Hamilton DW, Oates CJ, Hasanzadeh A, Mittler S. Migration of periodontal ligament fibroblasts on nanometric topographical patterns: influence of filopodia and focal adhesions on contact guidance. PLoS One. 2010 Dec 1;5(12): e15129.

4. Harrison S, Vavken P, Kevy S, Jacobson M, Zurakowski D, Murray MM. Platelet activation by collagen provides sustained release of anabolic cytokines. Am J Sports Med. 2011 Apr;39(4):729-34.

5. Y1lmaz S, Cakar G, Ipci SD. Platelet rich plasma in reconstructive periodontal therapy. InProgress in Molecular and Environmental Bioengineering-From Analysis and Modeling to Technology Applications 2011. InTech.

6. Sánchez-González DJ, Méndez-Bolaina E, Trejo-Bahena NI. Platelet-rich plasma peptides: key for regeneration. Int J Peptides. 2012;2012.

7. Fufa D, Shealy B, Jacobson M, Kevy S, Murray MM. Activation of platelet-rich plasma using soluble type I collagen. J Oral Maxillofac Surg. 2008 Apr 1;66(4):684-90.

8. Scanlon CS, Marchesan JT, Soehren S, Matsuo M, Kapila Y. Capturing the regenerative potential of periodontal ligament fibroblasts. J Stem Cells Regenerative Med. 2011;7(1):54.

9. Naik AR, Ramesh AV, Dwarkanath CD, Naik MS, Chinnappa AB. Use of autologous platelet rich plasma to treat gingival recession in esthetic periodontal surgery. J Indian Soc oPeriodontol. 2013 May; 17(3):345.

10.Parenteau-Bareil R, Gauvin R, Berthod F. Collagen-based biomaterials for tissue engineering applications. Materials. 2010 Mar 16;3(3):1863-87.

11.Li Z, Delaney MK, O'brien KA, Du X. Signaling during platelet adhesion and activation. Arterioscleros, Thromb, Vasc Bioy. 2010;30(12):2341-9. 